\section{An integrated stewardship model: antimicrobial, infection prevention and diagnostic (AID)}

\author{
Jan-Willem H Dik', Randy Poelman², Alexander W Friedrich,3, Prashant Nannan \\ Panday ${ }^{4}$, Jerome R Lo-Ten-Foe', Sander van Assen ${ }^{5}$, Julia EWC van Gemert-Pijnen ${ }^{6}$, \\ Hubert GM Niesters' ${ }^{2}$, Ron Hendrix ${ }^{1,7}$ \& Bhanu Sinha ${ }^{1}$
}

Considering the threat of antimicrobial resistance and the difficulties it entails in treating infections, it is necessary to cross borders and approach infection management in an integrated, multidisciplinary manner. We propose the antimicrobial, infection prevention and diagnostic stewardship model comprising three intertwined programs: antimicrobial, infection prevention and diagnostic stewardship, involving all stakeholders. The focus is a so-called 'theragnostics' approach. This leads to a personalized infection management plan, improving patient care and minimizing resistance development. Furthermore, it is important that healthcare regions nationally and internationally work together, ensuring that the patient (and microorganism) transfers will not cause problems in a neighboring institution. This antimicrobial, infection prevention and diagnostic stewardship model can serve as a blue print to implement innovative, integrative infection management.

First draft submitted: 22 July 2015; Accepted for publication: 14 August 2015;

Published online: 1 September 2015

Antimicrobial resistance is a growing public health threat, signaling the beginning of a postantimicrobial era [1]. Infections caused by multidrug-resistant organisms (MDROs) are associated with a significant deterioration of clinical outcomes. This includes an increased risk of mortality and morbidity, and it is associated with an increase in costs [2]. Recently, consensus has been achieved that the global community should act to limit these emerging problems as much as possible [1-3]. Emergence of antimicrobial resistance is strongly correlated with incorrect antimicrobial prescribing patterns and the lack of consistent diagnostic procedures to identify the pathogens involved, whether viral, bacterial or fungal. While antimicrobial medication has undoubtedly reduced mortality due to infections, resistance to these drugs renders them ineffective. To address these issues, healthcare institutions are implementing Antimicrobial Stewardship Programs (ASPs) to manage antimicrobial usage with the goal of improving patient outcomes, minimizing collateral damage and reducing the incidence of MDRO infections. They aim at treating patients according to the pathogens involved, based on a diagnostic strategy that ultimately keeps control on increasing expenditures in healthcare [4-6]. Participating in ASPs is mainly seen as a task of clinical microbiologists and/or infectious diseases specialists,

'Clinical Bacteriology Unit, Department of Medical Microbiology, University of Groningen, University Medical Center Groningen, Groningen, The Netherlands

${ }^{2}$ Clinical Virology Unit, Department of Medical Microbiology, University of Groningen, University Medical Center Groningen, Groningen, The Netherlands

${ }^{3}$ Infection Prevention Unit, Department of Medical Microbiology, University of Groningen, University Medical Center Groningen, Groningen, The Netherlands

${ }^{4}$ Department of Clinical Pharmacy, University of Groningen, University Medical Center Groningen, Groningen, The Netherlands ${ }^{5}$ Department of Internal Medicine, University of Groningen, University Medical Center Groningen, Groningen, The Netherlands ${ }^{6}$ Department of Psychology Health \& Technology, Faculty Behavioral, Management \& Social Sciences, University of Twente, Enschede, The Netherlands

${ }^{7}$ Certe Laboratory for Infectious Diseases, Groningen, The Netherlands

*Author for correspondence: Tel.: +31 503613 480; alex.friedrich@umcg.nl

\section{KEYWORDS}

- antimicrobial resistance - antimicrobial stewardship

- diagnostic stewardship

- infection management

- infection prevention stewardship • personalized treatment $\bullet$ point-of-impact testing 
together with (hospital) pharmacists. However, as patients move within the hospital, many more stakeholders are involved: bedside doctors, nurses and boards of directors and diagnostic laboratory directors who are responsible for providing financial resources. Furthermore, patients are not only moving within one particular hospital but also between different hospitals and other healthcare institutions as part of a comprehensive healthcare network. Therefore, infection management in a specific (part of an) institution will affect patients throughout the entire network [7,8]. Infection management is thus a responsibility of all stakeholders involved in this network. Additionally, recent developments in cross-border patient care have even extended these current healthcare networks to different countries, following the new EU directive on patients' rights in cross-border healthcare (directive 2011/24/EU) [9-11]. Since microbes will not adhere to any borders made by humans (i.e., departments, institutions or countries), we are forced to think and act in the same manner and closely collaborate in developing and implementing strategies that prevent patients from being colonized or infected with MDRO.

Cohen et al., recently advocated the use of a 'multifaceted 'omics' approach', to decrease the turn-around time of microbiological diagnostics [12]. We agree with this vision, but think this solution is part of a bigger picture. New diagnostic tools are important, but so is the interpretation and translation of the results into the day-to-day infection management. This requires cooperation of everyone involved in one large network.

We therefore strongly advocate collaboration of several disciplines within and between involved (healthcare) institutions, regionally and internationally, thereby crossing multiple borders. The main challenge is to develop an integrated system of not only ASPs, but also Infection Prevention Stewardship Programs (ISP) as well as Diagnostic Stewardship Programs (DSP), combined in an 'AID stewardship model'. These combined stewardship programs should be consultancy-based systems, involving and supporting stakeholders within the healthcare network (Figure 1), aiming at optimizing (laboratory) diagnostics, interpreting results and initiating correct and appropriate antimicrobial therapy (Figure 2). Furthermore, they act at the network level, aiding in taking the right infection control measures in order to provide a safe environment for patients and healthcare workers. Ultimately, this should also lead to more cost-effective healthcare in the mid-to-long term. In this view much can be learned from the theragnostic approach in cancer therapy, a holistic approach combining genetics, nuclear medicine and laboratory diagnostics guiding to adjust therapy [13]

Modern and rapid diagnostics should focus on individual patient care. Thus, from the viewpoint of individual patients, rapid identification of the causative agent, initiating optimal therapy and preventing the spread of highly infectious and pathogenic micro-organisms are crucial. A more classical approach divides the world into microbes, host and population and leads to a division between diagnostics, patient care and public health. This is particularly clear in countries where microbiology laboratories are located far away from patient care and even from clinical expertise. A stringent patient-oriented and personalized approach is needed where clinical consequences are directly dependent on timely generated microbiological (molecular) diagnostics. Consequently, in the field of antimicrobial therapy, appropriate and timely diagnostics need to be initiated before starting therapy. This will improve infection management on patient level by avoiding inadequate therapy and thus preventing collateral damage such as toxicity, driving antimicrobial resistance and spread of (MDR) organisms within the healthcare network.

To attain this goal of a theragnostic approach to infection management, we have developed a cross-border AID stewardship program, which is already partly implemented. Many are contributing to this integrated infection management system, each with a specific background and training. To facilitate this innovative and integral approach of infection management, the implementation of novel eHealth systems is essential as well as input of social sciences to take into account the influence of behavior [14-16]. Our developed program is an example of how the AID stewardship model could be implemented and can provide a blueprint or inspiration for others in the field of infection management and beyond.

\section{Antimicrobial stewardship}

Within the AID stewardship model, the ASP is based on the American (SHEA/IDSA) guidelines and the Dutch and German guidelines for ASPs [17-21]. It represents a program comprising various building blocks as shown in the master scheme (Figure 2). Key components of this 
scheme are: appropriate and timely microbiological diagnostics, calculated empirical therapy based on up-to-date regional/local epidemiology, close cooperation with the pharmacy department [20-22] and continuous clinical and proper financial outcome evaluations [6].

Our ASP program within the AID stewardship model especially focuses on a day-2 bundle with intervention taking place on the second day of antimicrobial therapy through face-to-face case audits by the antimicrobial stewardshipteam (A-Team) [22,23]. Triggered by an emailalert, an A-Team member will go to the ward and discuss the antimicrobial therapy with the bedside physician with a goal to improve and streamline the therapy using the by-then available diagnostics. This A-Team comprises clinical microbiologists, infectious diseases specialists and hospital pharmacists. They are supported by designated, trained doctors (link doctors) and nurses (link nurses) on every hospital ward. This bundle intervention aims for proactive discussions with prescribing doctors, leading to a consensus-driven intervention optimizing antimicrobial therapy, depending on patient status and the available diagnostic results. Early results are highly positive, both clinically and financially $[23,24]$. This highly interactive, face-to-face approach is dependent on the complexity of the patient's condition, whereby the more complex patients demand more sophisticated diagnostics and the expertise of (senior) specialists from different disciplines (Figure 1). This implies crossing traditional borders between disciplines and combining the knowledge and expertise of the treating physicians and the A-Team members optimally for the benefit of the individual patient and the healthcare institution.

An important, and in practice often somewhat neglected, area is the optimization and personalization of therapy. This should take into account basic patient factors such as preliminary diagnosis, compartment of the infection, bodyweight, pharmacokinetic aspects (including organ dysfunction and current volume of distribution, among others), pharmacodynamic characteristics of the drugs (e.g., killing activity, among others) and characteristics of the microorganisms (most relevantly the MIC). An integration of these factors can be performed using pharmacokinetic and pharmacodynamic (PK/ PD) data and models. This approach can be used to optimize empiric therapy (based on population data for both patients and microorganisms),

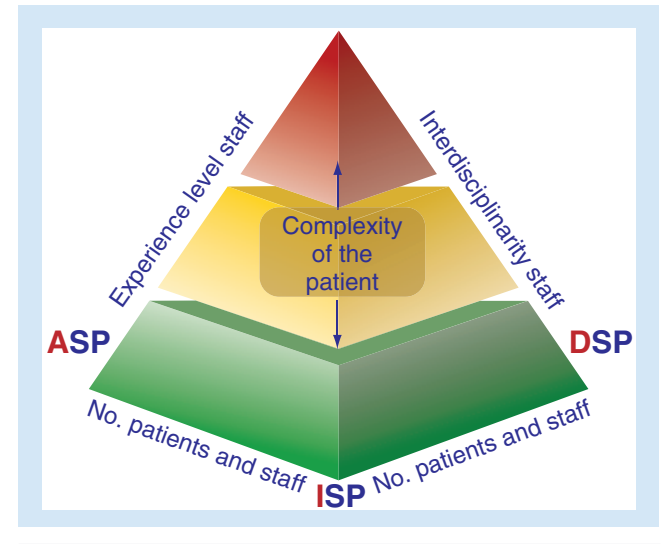

Figure 1. Multistakeholder platform of the antimicrobial, infection prevention and diagnostic stewardship model. Pyramid platform showing the interdisciplinary stakeholder connections between the ASP, ISP and DSP within the antimicrobial, infection prevention and diagnostic stewardship model. This model represents the complexity of the patients (green for low complex, orange for intermediate complex and red for high complex) that corresponds with number of patients and treating staff (width of the pyramid) and the experience level of the treating staff (height of the pyramid). The more complex a patient, the more he/she requires experienced specialists from multiple disciplines supplemented with correct, on-time performed diagnostics and eHealth tools. This, together, is needed to adequately deal with the specific infectious problems, whereby the complexity of the patient is not a fixed label, but a continuous changing state which varies over time.

ASP: Antimicrobial Stewardship Program;

DSP: Diagnostic Stewardship Program; ISP: Infection Prevention Stewardship Program.

as well as personalizing therapy for individual patients over time. Especially for the latter aspect, appropriate therapeutic drug monitoring (TDM) needs to be implemented. When integrating all available data, both optimizing the effect of antimicrobial therapy as well as limiting collateral damage (toxicity and emergence of resistance) can be addressed.

Before starting the development and implementation of an ASP, it is important to assess the already implemented activities, as well as the specific requirements of the healthcare institution. Therefore, we have developed an ASP maturity model approach, which can be used for such an assessment. This will help tailor an ASP 


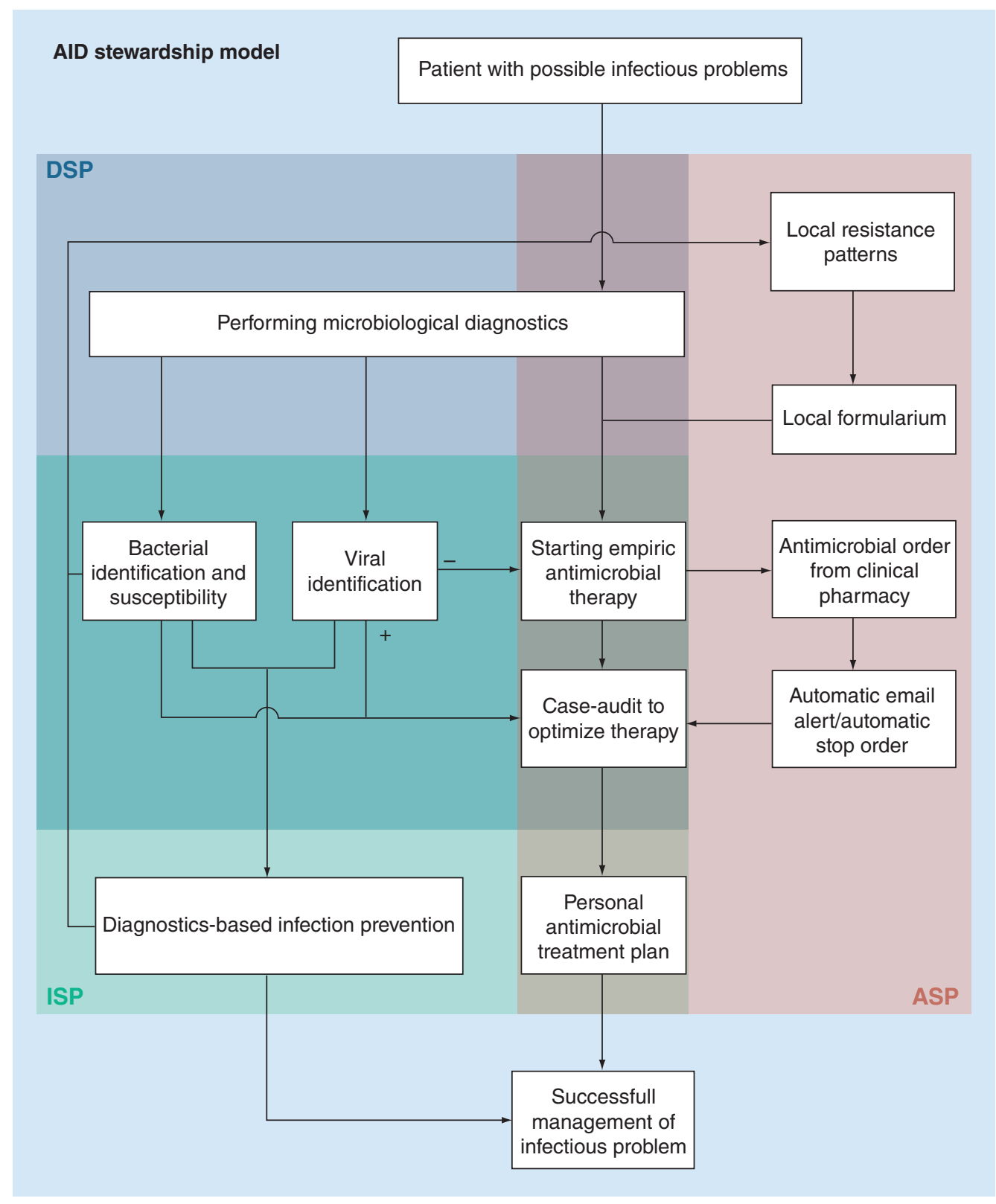

Figure 2. Master scheme antimicrobial, infection prevention and diagnostic stewardship model. Flow chart that depicts the path of care of a patient from top to bottom, surrounded by (several of) the building blocks of the three different, but supplemental, stewardship programs and how they are intertwined. Notice the overlap, thereby showing the necessity of all three stewardship programs and integrative nature of the model.

AID: Antimicrobial, infection prevention and diagnostic; ASP: Antimicrobial Stewardship Program; DSP: Diagnostic Stewardship Program; ISP: Infection Prevention Stewardship Program.

program to the specific needs of the healthcare institution making it easier to integrate within an overarching model such as the AID model [4]. Furthermore, preparing for a future with more cross-border patient movements, it is vital to also facilitate cross-border capacity building for the development of A-Teams in the clinical practice. This fosters collaboration and knowledge transfers between science, health and small/ medium enterprises ultimately leading to new innovative eHealth technologies from an enduser involvement perspective [14-16]. For the support of activities of the A-Teams, we already have developed several of these eHealth tools, notably 
mobile applications and automatic e-mail alerts, in order to facilitate easy access to diagnostic and therapeutic data, which significantly improved the decision-making processes [25,26]. However, more eHealth tools are available, most notably different clinical discussion support systems (CDSSs), which can aid and support the prescriber and/or the A-Team in optimizing antimicrobial therapy [27]. It is important to create an ICT-structure that can be used to transfer patient data safely and confidently. This is not yet implemented for microbiological diagnostics. However, within our healthcare region this is for example already achieved for radiographic data.

\section{Infection prevention stewardship}

It is vital that infection control and prevention measures are integrated into this unified program to improve overall infection management. Without the proper infection prevention measures, other interventions such as ASPs and DSPs will not yield the optimal effect. Within the AID stewardship model, infection prevention stewardship entails early detection and close surveillance of MDROs, as well as an adequate rapid reaction to every possible transmission (Figure 2). This task is dependent on easy access to rapid microbiological diagnostics for both direct patient care and surveillance purposes. Therefore, our units of infection control and prevention and medical microbiology (bacteriology, virology, mycology and parasitology) are organized within one single department for maximal collaboration and cooperation and work together closely with the internal medicine department and the hospital pharmacy. Together, they are responsible for, for example, hand hygiene guidelines and audits, isolation measures and patient-specific consultations and hospital-wide infrastructure (e.g., contribution to in-hospital guidelines for infections and the antimicrobial formularium). The success story of the containment of MRSA within The Netherlands by a search-and-destroy strategy is an example of the substantial positive effect of close cooperation between clinical microbiology and infection prevention specialists [28]. This applies to bacteria as well as viruses where similar screening programs are also beneficial [29-31]. Continuous communication with other ISP consultants within the healthcare network and developing and updating unified guidelines at the regional level is important $[8,32,33]$. In ISP, the use of eHealth technology, developed in a multidisciplinary environment by medical experts together with eHealth experts, has considerable potential to facilitate these work processes [14,16]. For example, it has been shown that web-based guidelines are suboptimally used by nurses [34]. With the aid of user-centered and persuasive eHealth systems, the adherence to guidelines improved and errors in using them reduced significantly [34]. These systems were developed as part of MRSA-net [35] and EurSafety Health-net [36] in the DutchGerman Euregio, exemplifying the potential of eHealth for ISPs [25,37].

\section{Diagnostic stewardship}

To provide the optimal therapy for individual patients but also for infection control and prevention purposes, it is essential that state-ofthe-art diagnostics are performed timely, before initiating antimicrobial therapy. Diagnostics must be appropriate for the individual patient, target all pathogens causing acute infections and detect colonization and/or infection. Helping individual physicians in selecting and interpreting diagnostic tests on the appropriate clinical specimens is the major goal of diagnostic stewardship. To be most effective, these diagnostic tests should provide relevant clinical data as soon as possible, but for sure within the first hours of admission (viral) or the first 24-48 h of admission (bacterial and fungal). Molecular diagnostics can largely meet these requirements. With new point-of-care (or point-of-impact) assays (e.g., testing different biomarkers) becoming available, the turnaround time for an increasing number of viral and bacterial/fungal pathogens can be reduced to less than $2 \mathrm{~h}$, supporting clinical decision-making. An important issue is furthermore supporting a noninfectious differential diagnosis for certain conditions if appropriate diagnostics rapidly yield negative results. Similar as the theragnostics approach in oncology, this should ultimately lead to a more personalized infection management plan for the patient, whereby diagnostics, therapy and infection prevention are integrated.

The use of innovative methods (e.g., next-generation sequencing) is an exciting evolving field within clinical microbiology and infection control and is advocated by more as one of the solutions for antimicrobial resistance [12]. It fits within the theragnostic approach to treatment and, therefore, innovative point-of-impact technologies and real-time sequencing tools are already put into use within the AID stewardship model 
and will be fully implemented in standard daily care in the near future $[31,38]$. We expected that also novel comprehensive diagnostic tools will provide results much faster and more accurately, as already shown to be the case in combined virological and bacteriological diagnostics (e.g., multiplex molecular tests) $[39,40]$. This can provide the basis for better and more personalized therapy for patients, contributing to an optimized use of antimicrobials, surveillance and infection control, all leading to a more theragnostic approach of infection management. Of course, that does not disqualify the use of conventional, culture-based diagnostics like conventional blood, urine sputum and other cultures combined with susceptibility testing. Bacterial cultures for example are still an effective and relatively cheap diagnostic tool for the diagnosis of infections/colonization. In addition, they are the only means to assist in storage and typing of specific isolates. However, the turnaround times of these tests often are too long to be useful in the early stages of treatment. Especially in regions where diagnostic facilities are situated far away from the actual point of patient care, logistics can negatively impact the turnaround time. Making these tools available in the near proximity of patient care will significantly reduce turnaround times.

With the implementation of rapid point-ofimpact technologies, subsequent rapid decisionmaking will be beneficial for the optimal use of resources (for instance bed management and isolation room capacity). These diagnostic assays and next-generation diagnostics are mostly based on molecular technologies and are therefore more expensive compared with classical culture-based methodology. They are however faster, delivering results within hours, thereby enabling a theragnostic approach for infection management. A proper cost-effectiveness study can provide the validation for the implementation of these tests. However, from a managerial point of view and to support health economical decision-making, the so-called '€hr concept' can easily make turnaround times visible in relation to the overall costs (such as costs for unnecessary isolation). By multiplying costs (€) and turnaround time $(\mathrm{h})$, it provides a quick, understandable figure, assuming that quality remains high and therefore equals one. This provides the basis of the diagnostics needed for the AID stewardship (Figure 3).

Integrated stewardship: crossing multiple

\section{borders}

In our view, infection management cannot be adequately performed by one single doctor, by one medical specialty, by one hospital or even by one country. Infection management in healthcare networks is based on an interdisciplinary and inter-regional approach (Figure 1). Movement of patients within and between healthcare institutions entails movement of microorganisms within and to other institutes as well. For this reason, we have developed an integrated AID stewardship model in close cooperation with healthcare institutions and diagnostic laboratories within the region.

Due to the regional nature, this program is part of a larger Euregional collaboration between The Netherlands and Germany, initially started on a relatively small scale with the MRSA-net project. It has, in the meanwhile, been extended to the EurSafety Health-net project [36] covering the complete Dutch-German border region (>8 million inhabitants). Over the years, this resulted in an intense collaboration of more than 115 acute care hospitals, more than 200 longterm care facilities and a large array of healthcare institutions, working together on infection management. Optimizing patient care and infection control by building networks of stakeholders, knowledge transfer and consolidating the best of both worlds has always been the primary focus. Within this cross-border network, the focus lies on ASP, ISP and DSP. In this approach, eHealth contributes significantly to the different goals in these projects. Among many other results, this has led to tools for clear and easy presentation of MRSA protocols [17,34,37], but also middleware solutions to improve molecular diagnostics like FlowG [41]. In order to facilitate the implementation of the AID stewardship model, the EurSafety Health-net project has led to various other applications that were developed and can be used on-site by infection experts, healthcare providers and patients, implementing eHealth in daily cross-border practice $[15,25,27,40]$.

\section{Conclusion}

In our view, managing antimicrobial infections can only be achieved by a holistic approach based on the theragnostic principles as exemplified by the AID stewardship model, with the implementation of timely (innovate) diagnostics, woven into the treatment management plan and infection prevention. When combining ASP, ISP and DSP, one is able to target the complete 
healthcare network, which can be supported by health economics, social sciences, technological sciences and by using eHealth technologies.

This technology is crucial to support multidisciplinary and cross-border infection management, to share knowledge and to support healthcare workers and infection specialists with easy accessible and user-friendly instructions for stewardship programs. First, to share knowledge and thoughts about integrated AID stewardship in healthcare; second to provide systems to support staff in implementing stewardship programs in daily work; third to guide the implementation process related to local situations and global guidelines for safe work and reduction of antimicrobial resistance rates. The University of Twente (Enschede, The Netherlands) develops the ecosystem for novel technologies that address these questions. In the EurSafety Health-net project, several technologies have already been developed to support the administration of antimicrobials [25], to improve surveillance of infections [26], and to implement stewardship programs [4]. These technologies are created in co-design with the end users (e.g., nurses, physicians and infection prevention specialists). Key stakeholders in the crossborder network need to be involved and contribute appropriately to the system to guarantee the model will be effectively implementable $[15,42,43]$.

Within this model, it is vital to work together and to utilize expertise of various stakeholders and organizations to intervene at various time points in infection management. The three different stewardship aspects are highly intertwined and must be implemented adequately. Especially in regions with higher resistance rates, this integration is even more important in order to control the situation and work toward an improvement. The AID stewardship model is in that respect generally applicable because the main focus point is the integration of the three stewardship programs. The specific actions performed within these programs depend on the setting of the healthcare institution. Implementing a monovalent ASP, as advocated in several countries, is of limited use without the implementation of appropriate and more sophisticated diagnostics close patient care and vice versa. Since hospitals are part of comprehensive healthcare networks, it is not helpful if one hospital has implemented a complete stewardship program, but the hospital next door has not made proper, similar arrangements.

Therefore, we encourage stakeholders to cross borders and organize patient-centered infection

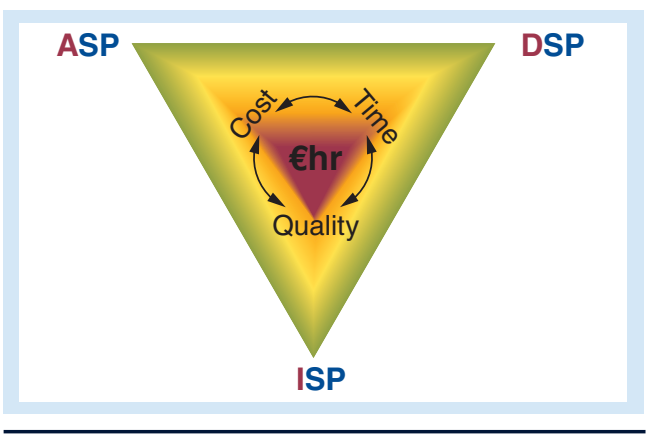

Figure 3. €hr concept within the antimicrobial, infection prevention and diagnostic stewardship model. A diagram showing a top-view of Figure 1. Diagnostics should be present to provide an integrative and effective stewardship program such as antimicrobial, infection prevention and diagnostic. From a managerial point of view, the effectiveness of these diagnostics can be described with the €hr concept, visualizing the most important aspects: turnaround time (h) and costs $(€)$, assuming that quality remains high and therefore equals one. ASP: Antimicrobial Stewardship Program; DSP: Diagnostic Stewardship Program; ISP: Infection Prevention Stewardship Program.

management in a theragnostic, multifaceted, multidisciplinary, inter-regional approach to counteract antimicrobial resistance problems. In this manner, smaller institutions without extensive resources can benefit from academic centers and these centers in turn benefit with the referral of patients. The AID stewardship model that we are enrolling is an example of such an approach, which could serve as a blueprint in the field of infection management worldwide.

\section{Future perspective}

An increasing amount of microbiology data is becoming available for clinicians to be used when treating patients, including genomic data and information on the potential pathogen. Furthermore, it will get easier to link already existing data from different databases to provide a real-time up-to-date overview of the patient and his treatment (such as MIC values and $\mathrm{PD} / \mathrm{PK}$ information), that clinician can use. Clinical decision support systems are already becoming available that can help the clinician in interpreting this increasing amount of data. These programs are expected to become smarter and more comprehensive in the near future. All 
this should hopefully lead to a situation where personalized treatment can be started right away or at least within the first hours after a patient enters the hospital with a (suspected) infectious problem. This will be facilitated by the use of novel diagnostic technologies (e.g. third/fourth generation sequencing and spectroscopy) and smart decision support systems. ASPs can utilize this data to ensure optimal treatment at all times, acting upon PK/PD data in real time. This should limit the amount of inadequately prescribed antimicrobials, thereby minimizing resistance pressure and collateral damage to patients. It would optimize the complete infection management process, thus providing better and more (cost-)efficient patient care. Furthermore, these data could facilitate optimized infection prevention within the healthcare region, by providing real-time patient and pathogen characteristics to all involved healthcare institutions. It is vital that stakeholders cross borders and start collaborating as soon as

\section{EXECUTIVE SUMMARY}

- Antimicrobial resistance is a growing worldwide public health threat and the result of suboptimal infection management.

- We advocate more collaboration between all stakeholders within and between (healthcare) institutions, regionally and internationally.

- The here proposed antimicrobial, infection prevention and diagnostic (AID) stewardship model is an example of such a strong collaboration.

- The AID stewardship model is a theragnostic approach to infection management addressing antimicrobial, infection prevention and Diagnostic Stewardship Programs.

\section{Antimicrobial stewardship}

- Key components are appropriate, timely microbiological diagnostics, calculated empirical therapy, close cooperation with the pharmacy department and continuous clinical and financial outcome evaluations with a focus on a day-2 bundle of audit/feedback.

- Pharmacokinetic and pharmacodynamic data are used to further improve and personalize antimicrobial treatment.

Infection prevention stewardship

- Infection Prevention Stewardship Program entails early detection and close surveillance of multidrug-resistant organisms, as well as an adequate rapid reaction to every possible transmission.

- Continuous communication within the healthcare region and unification of guidelines is important.

\section{Diagnostic stewardship}

- For personalized treatment and for infection control and prevention purposes, state-of-the-art diagnostics should be performed timely, before initiating antimicrobial therapy.

- Diagnostics must be appropriate and detect colonization and/or infection within 24-48 h of admission.

\section{Integrated stewardship: crossing multiple borders}

- Infection management in healthcare networks should be based on an interdisciplinary and inter-regional approach of all stakeholders.

- The AID stewardship model supports this approach and is part of a larger Euregional collaboration between The Netherlands and Germany.

- This international collaboration led to multiple eHealth applications that facilitate the AID stewardship model.

\section{Conclusion \& future perspective}

- Managing antimicrobial infections can only be achieved by a holistic approach based on the theragnostic principles as exemplified by the AID stewardship model.

- eHealth technology is crucial to support multidisciplinary, cross-border infection management.

- We encourage stakeholders to cross borders and organize patient-centered infection management in a theragnostic, multifaceted, multidisciplinary, inter-regional approach to counteract antimicrobial resistance problems. 
possible, in order to provide an integrative infection management such as the proposed integrative stewardship model. By an integrative stewardship approach, such as our proposed AID model, healthcare institutions will immediately be streamlining the infection management process. This will form a foundation of stakeholder collaborations that are necessary to utilize the upcoming flow of data and integrate these data in day-to-day patient care.

\section{Acknowledgements}

The authors would like to thank all personnel involved in the AID stewardship model and infection management in general, especially the A-Team members, Link docs, Link nurses, infection prevention specialists and colleagues from the contributing institutions. Furthermore, we would like to thank Nienke de Jong of the University of Twente for critically reviewing the eHealth sections, as well as Maarten Postma from the pharmacoeconomics department of the University of Groningen for fruitful discussions during the process of developing the AID stewardship model.

\section{References}

Papers of special note have been highlighted as: - of interest

1 World Health Organization. The evolving threat of antimicrobial resistance - options for action.

http://whqlibdoc.who.int/publications

2 Smith R, Coast J. The true cost of antimicrobial resistance. BMJ 346, f1493 (2013).

3 Laxminarayan R, Duse A, Wattal C et al. Antibiotic resistance - the need for global solutions. Lancet Infect. Dis. 13(12), 1057-1098 (2013).

- A very complete overview on antibiotic resistance, the treatment it entails and actions that should be undertaken.

4 van Limburg M, Sinha B, Lo-Ten-Foe JR, van Gemert-Pijnen JEWC. Evaluation of early implementations of antibiotic stewardship program initiatives in nine Dutch hospitals. Antimicrob. Resist. Infect. Control 3(1), 33 (2014).

5 Davey P, Brown E, Charani E et al. Interventions to improve antibiotic prescribing practices for hospital inpatients. Cochrane Database Syst. Rev. 4, CD003543 (2013).

- The most comprehensive review available regarding antimicrobial stewardship.

6 Dik JH, Vemer P, Friedrich AW et al. Financial evaluations of antibiotic

\section{Financial \& competing interests disclosure}

Part of this work was supported by the European Union, the German states of North Rhine-Westphalia and Lower Saxony, and the Dutch provinces Overijssel, Gelderland and Limburg via the EurSafety Health-net project (Interreg IVa III-1-01=073). B Sinha has received a travel grant co-funded by Pfizer/Wyeth, and worked on projects in co-operation with Pathogenica Life Technologies, and Copan, the other authors have no possible conflicts of interest regarding this article. The authors have no other relevant affiliations or financial involvement with any organization or entity with a financial interest in or financial conflict with the subject matter or materials discussed in the manuscript apart from those disclosed.

No writing assistance was utilized in the production of this manuscript.

\section{Open access}

This work is licensed under the Creative Commons Attribution-NonCommercial 3.0 Unported License. To view a copy of this license, visit http://creativecommons.org/ licenses/by-nc-nd/3.0/

stewardship programs - a systematic review. Front. Microbiol. 6, 317 (2015).

7 Donker T, Wallinga J, Grundmann $\mathrm{H}$. Patient referral patterns and the spread of hospital-acquired infections through national healthcare networks. PLoS Comput. Biol. 6(3), e1000715 (2010).

8 Ciccolini M, Donker T, Köck RR et al. Infection prevention in a connected world: the case for a regional approach. Int. J. Med. Microbiol. 303(6-7), 380-387 (2013).

9 Friedrich AW, Daniels Haardt I, Köck RR et al. EUREGIO MRSA-net Twente/ Münsterland - a Dutch-German cross-border network for the prevention and control of infections caused by methicillin-resistant Staphylococcus aureus. Euro. Surveill. 13(35), pii:18965 (2008).

10 Deurenberg RH, Nulens E, Valvatne $\mathrm{H}$ et al. Cross-border dissemination of methicillinresistant Staphylococcus aureus, Euregio Meuse-Rhin region. Emerg. Infect. Dis. 15(5),727-734 (2009).

11 Köck RR, Brakensiek L, Mellmann A et al. Cross-border comparison of the admission prevalence and clonal structure of meticillinresistant Staphylococcus aureus. J. Hosp. Infect. 71(4), 320-326 (2009).

12 Cohen A, Bont L, Engelhard D et al. A multifacted 'omics' approach for addressing the challenge of antimicrobial resistance. Future Microbiol. 10(3), 365-376 (2015).
13 Pene F, Courtine E, Cariou A, Mira JP. Toward theragnostics. Crit. Care Med. 37(1 Suppl.), S50-S58 (2009).

14 van Gemert-Pijnen JEWC, Nijland N, van Limburg $\mathrm{M}$ et al. A holistic framework to improve the uptake and impact of eHealth technologies. J. Med. Internet Res. 13(4), e111 (2011).

15 Janes CR, Corbett KK, Jones JH, Trostle J. Emerging infectious diseases: the role of social sciences. Lancet 380(9857), 1884-1886 (2012).

16 van Gemert-Pijnen JE, Peters O, Ossebaard HC. Improving eHealth. Eleven International Publishing, Den Haag, The Netherlands (2013).

17 Dellit T, Owens R, McGowan J et al. Infectious Diseases Society of America and the Society for Healthcare Epidemiology of America guidelines for developing an institutional program to enhance antimicrobial stewardship. Clin. Infect. Dis. 44(2), 159-177 (2007).

18 With de K, Allerberger F, Amann S et al. S3-Leitlinie. Strategien zur Sicherung rationaler Antibiotika-Anwendung im Krankenhaus. www.awmf.org/uploads/tx_szleitlinien

19 SWAB. [The quality of antibiotic policy in The Netherlands].

www.swab.nl/swab/cms3.nsf/uploads

20 Pulcini C, Defres S, Aggarwal I, Nathwani D, Davey P. Design of a 'day 3 bundle' to improve 
the reassessment of inpatient empirical antibiotic prescriptions. J. Antimicrob. Chemother. 61(6), 1384-1388 (2008).

21 Cooke FJ, Holmes AH. The missing care bundle: antibiotic prescribing in hospitals. Int. J. Antimicrob. Agents 30 (1), 25-29 (2007).

22 Lo-Ten-Foe JR, Sinha B, Wilting KR, Veenstra-Kyuchukova Y, Panday PN, Hendrix R. [Bedside consultation by a multidisciplinary antibiotics team: an Antibiotic Stewardship Programme at UMCG]. Ned Tijdschr Geneeskd. 158(5), A6795 (2014).

23 Dik JH, Sinha B, Lo-Ten-Foe JR et al. Cost-minimization model of a multidisciplinary Antibiotic StewardshipTeam based on a successful implementation on a urology ward of an academic hospital. PLoS ONE 10(5), e0126106 (2015).

24 Dik JH, Hendrix R, Lo-Ten-Foe JR et al. Automatic day-2 intervention by a multidisciplinary antimicrobial stewardshipteam leads to multiple positive effects. Front. Microbiol. 3(6), 546 (2015).

25 Wentzel J, van Velzen L, van Limburg M et al. Participatory eHealth development to support nurses in antimicrobial stewardship. BMC Med. Inform. Decis. 14(1), 45 (2014).

26 Beerlage-de Jong N, Eikelenboom-Boskamp A, Voss A, Sanderman R, van Gemert-Pijnen JEWC. Combining user-centered design with the persuasive systems design model: the development process of a web-based registration and monitoring system for healthcareassociated infections in nursing homes. Int. J. Adv. Life Sci. 6(4), 262-271 (2014).

27 Beaulieu J, Fortin R, Palmisciano L, Elsaid K, Collins C. Enhancing clinical decision support to improve appropriate antimicrobial use. Am. J. Health Syst. Pharm. 70 (13), 1103-1104 (2013).
28 Köck RR, Becker K, Cookson B et al. Systematic literature analysis and review of targeted preventive measures to limit healthcare-associated infections by meticillinresistant Staphylococcus aureus. Euro. Surveill. 19(37), pii:20902 (2014).

29 Poelman R, Schölvinck EH, Borger R, Niesters HG, van Leer-Buter C. The emergence of enterovirus D68 in a Dutch University Medical Center and the necessity for routinely screening for respiratory viruses. J. Clin. Virol. 62, 1-5 (2015).

30 Poelman R, Schuffenecker I, Van Leer-Buter C, Josset L, Niesters HGM, Lina B, on behalf of the ESCV-ECDC EV-D68 study group. European surveillance for enterovirus D68 during the emerging North-American outbreak in 2014. J. Clin. Virol. 71, 1-9 (2015).

31 Rahamat-Langendoen JC, Lokate M, Schölvinck EH, Friedrich AW, Niesters HG. Rapid detection of a norovirus pseudo-outbreak by using real-time sequence based information. J. Clin. Virol. 58(1), 245-248 (2013).

32 Müller J, Voss A, Köck RR et al. Cross-border comparison of the Dutch and German guidelines on multidrug-resistant Gramnegative microorganisms. Antimicrob. Resist. Infect. Control 4, 7 (2015).

33 Verhoeven F, van Gemert-Pijnen L, Hendrix MGR et al. Development of a web-based learning tool to enhance healthcare workers' knowledge, attitude, and risk perception about safe work practices concerning meticillin-resistant Staphylococcus aureus. Clin. Microbiol. Infect. 14(S7), 663 (2013).

34 Verhoeven F, Steehouder MF, Hendrix MGR, van Gemert-Pijnen JEWC. How nurses seek and evaluate clinical guidelines on the Internet. J. Adv. Nurs. 66(1), 114-127 (2010)
35 MRSA-Net. www.mrsa-net.nl

36 EurSafety Health-Net. www.eursafety.eu

37 Jurke A, Köck RR, Becker K et al. Reduction of the nosocomial meticillin-resistant Staphylococcus aureus incidence density by a region-wide search and follow-strategy in forty German hospitals of the EUREGIO, 2009 to 2011. Euro. Surveill. 18(36), pii:20860 (2013).

38 Zhou K, Ferdous M, de Boer RF et al. The mosaic genome structure and phylogeny of Shiga toxin-producing Escherichia coli O104:H4 is driven by short-term adaptation. Clin. Microbiol. Infect. 21(5), 468.e7-468.e18 (2014).

39 Popowitch EB, O’Neill SS, Miller MB. Comparison of the Biofire FilmArray RP, Genmark eSensor RVP, Luminex xTAG RVPv1, and Luminex xTAG RVP fast multiplex assays for detection of respiratory viruses. J. Clin. Microbiol. 51(5), 1528-1533 (2013).

40 Rogers BB, Shankar P, Jerris RC et al. Impact of a rapid respiratory panel test on patient outcomes. Arch. Pathol. Lab. Med. 139(5), 636-641 (2015).

41 FlowG - FlowGroningen MiddleWare. www.flowg.nl

42 van Limburg M, van Gemert-Pijnen JEWC, Nijland N, Ossebaard HC, Hendrix R, Seydel ER. Why business modeling is crucial in the development of eHealth technologies. J. Med. Internet Res. 13(4), e124 (2011).

43 Wentzel MJ, de Jong N, Karreman J, van Gemert-Pijnen JEWC. Implementation of MRSA infection prevention and control measures. What works in practice? In: Infection Control-Updates. Sudhaker C (Ed.). InTech, Reijka, Croatia (2012). 